cific procoagulants, whose concentration and effects are more prominent close to their site of origin. Both mechanisms have been demonstrated in patients with adenocarcinoma of the pancreas. ${ }^{2}$ However, reports of thrombosis in patients with mesothelioma are much rarer than in patients with adenocarcinoma of the pancreas or lung, for example, which suggests that the first mechanism is the more likely in the case we described; ${ }^{1}$ the chest CT shown in our report is consistent with this hypothesis. The surgery report did not allude to the state of the veins, and, unfortunately, ${ }^{3}$ an autopsy was not authorized. As for our description of Virchow's triad, use of the word "epithelial" was a typographic error, and the text should have referred to "endothelial damage" (on page 465 , third column).

The case reported by Mehlika Isildak and associates is an additional vivid reminder that cancer-associated thrombosis can affect veins at almost any site; it also emphasizes the greater risk with more advanced disease. ${ }^{1}$ However, thrombotic complications in mesothelioma remain an unusual occurrence in both early ${ }^{4}$ and advanced disease. Interleukin 6 (IL-6) may indeed be produced by mesothelioma and other tumours. ${ }^{5} \mathrm{It}$ affects not only the number of platelets but, more important, their function. Platelets responding to IL-6 have increased sensitivity to activation by thrombin and increased procoagulant activity, which may be further enhanced by the elevated levels of fibrinogen and plasminogen activator inhibitor (which suppresses fibrinolysis) caused by IL-6 and other inflammatory mediators. ${ }^{6}$ The exact relevance of these observations to thromboembolism in vivo remains unproven.

\section{Ami Schattner}

Hebrew University

Hadassah Medical School

Jerusalem, Israel

\section{References}

1. Schattner A, Lugassy G, Klepfish A. Thromboembolism in pancreatic cancer. Pathogenesis, manifestations and management. In: Columbus F, editor. Progress in pancreatic cancer research. New York: Nova Science Publishers. In press.
2. Schattner A, Kozack N. A 47-year-old man with mesothelioma and neck swelling. CMAf 2004; 170(4):465.

3. Lundberg GD. Low-tech autopsies in the era of high-tech medicine. Continued value for quality assurance and patient safety. 7AMA 1998;280: 1273-4.

4. Sorensen HT, Mellemkjaer L, Steffensen FH, Olsen JH, Nielsen GL. The risk of a diagnosis of cancer after primary deep venous thrombosis or pulmonary embolism. N Engl 7 Med 1998; 338:1169-73.

5. Suzuki S, Tanaka K, Nogawa S, Umezawa A, Hata J, Fukuuchi Y. Expression of interleukin-6 in cerebral neurons and ovarian cancer tissue in Trousseau syndrome. Clin Neuropathol 2002;21: 232-5.

6. Esmon CT. Possible involvement of cytokines in diffuse intravascular coagulation and thrombosis. Baillieres Best Pract Res Clin Haematol 1999;12: 343-59.

DOI:10.1503/cmaj.1040715

\section{Kickbacks and self-referral}

S ujit Choudhry and associates ${ }^{1}$ raise S some excellent points in their discussion of the unethical nature of physician kickbacks and physician selfreferral. An additional related behaviour is on the horizon for physicians in many provinces.

Electronic medical records and clinical management systems are now being promoted by federal and provincial authorities as a valuable component of care. One of the many functions currently being touted as desirable in a clinical management system is direct communication between the prescribing doctor and the patient's pharmacy for both new and repeat prescriptions.

For the vast majority of prescribing physicians and pharmacists, the ability to send a prescription to a pharmacy "at the touch of a button" will be a welcome relief from repetitive, illegible, hand-written prescriptions. For a few doctors and pharmacists, this function will represent an opportunity for hidden financial gain (through a kickback).

Time could be well spent asking questions about direct connections between a prescribing physician and a pharmacy. Provincial regulating authorities for both pharmacists and physicians should examine the risks and benefits of this functionality. They should also determine what reporting and control mechanisms are needed to minimize the temptation of a destructive conflict of interest.

\section{George Southey}

Lead Physician

Dorval Medical Associations Family

Health Network

Oakville, Ont.

\section{Reference}

1. Choudhry S, Choudhry NK, Brown AD. Unregulated private markets for health care in Canada? Rules of professional misconduct, physician kickbacks and physician self-referral [editorial]. CMA7 2004;170(7):1115-8.

Competing interests: None declared.

DOI:10.1503/cmaj.1040548

S ujit Choudhry and colleagues ${ }^{1}$ are $S$ to be congratulated for raising the troubling issues of physician kickbacks and self-referrals. ${ }^{1}$ The Medical Reform Group, of which I am a member, agrees that these practices are ethically dubious and ought to be much more closely regulated.

One simple way to limit kickbacks and self-referrals would be to ban investor-owned independent health facilities from operating within the publicly funded health care system. Kickbacks and self-referrals exist chiefly to increase profit. Disallow profit, and these practices would probably wither away.

There is another reason to consider banning investor-owned independent health facilities: quality. In the United States at least, investor-owned hospitals $^{2}$ and dialysis centres ${ }^{3}$ are associated with higher mortality rates than private, nonprofit facilities. Similarly, in a study comparing for-profit and nonprofit health maintenance organizations in the United States, the nonprofit organizations outperformed the for-profit ones on all of the 14 quality-of-care indicators that were assessed. ${ }^{4}$ In the Canadian context, for-profit independent health facilities are most common in the diagnostic services (e.g., laboratory testing and imaging) and rehabilitation (e.g., physiotherapy) sectors. Given the US data (there are none from Canada), there is no reason to assume that the services they provide are as good as those provided by nonprofit operators. 